\title{
JANVIER-FÉVRIER 1984
}

66॰ ANNÉE - N• 745


de la

croix-rouge

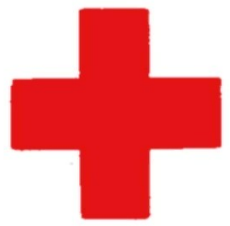

INTER ARMA CARITAS

GENEVE

COMITÉ INTERNATIONAL DE LA CROIX-ROUGE FONDÉ EN 1863 


\title{
COMITÉ INTERNATIONAL DE LA CROIX-ROUGE
}

MM. ALEXANDRE HAY, avocat, ancien directeur général de la Banque nationalo suisse, président (membre depuis 1975)

MAURICE AUBERT, docteur en droit, vice-président (1979)

VICTOR H. UMBRICHT, docteur en droit, administrateur, vice-président (1970)

JEAN PICTET, docteur en droit, ancien vice-président du CICR (1967)

M" DENISE BINDSCHEDLER-ROBERT, docteur en droit, professeur a l'Institut universitaire de hautes études internationales, Genève, juge à la Cour européenne des droits de l'homme (1967)

MM. JACQUES F. DE ROUGEMONT, docteur en médecine (1967)

GILBERT ETIENNE, professeur à l'Institut universitaire de hautes études internationales et à l'Institut universitaire d'études du développement, Genève (1973)

ULRICH MIDDENDORP, docteur en médecine, chef de la clinique chirurgicale de l'Hòpital cantonal, Winterthour (1973)

$M^{\text {me }}$ MARION BOVÉ-ROTHENBACH, docteur en sociologie (1973)

MM. HENRY HUGUENIN, banquier (1974)

RICHARD PESTALOZZI, docteur en droit, ancien vice-président du CICR (1977)

ATHOS GALLINO, docteur en médecine, maire de Bellinzone (1977)

ROBERT KOHLER, docteur ès sciences économiques (1977)

RUDOLF JÄCKLI, docteur ès sciences, (1979)

$M^{\text {He }}$ ANDREEE WEITZEL, ancien chef du service complémentaire féminin au Département militaire fédéral, vice-présidente de la Commission nationale suisse pour l'Unesco (1979)

MM. OLIVIER LONG, docteur en droit et docteur ès sciences politiques, ambassadeur, ancien directeur général du GATT (1980)

DIETRICH SCHINDLER, docteur en droit, professeur à l'Universitê de Zurich (1961-1973; 1980)

HANS HAUG, docteur en droit, professeur à l'Ecole des hautes études économiques et sociales de Saint-Gall, ancien président de la Croix-Rouge suisse (1983)

PETER ARBENZ, licencié ès sciences politiques, membre du Comité central d'Helvetas, conseiller municipal de Winterthur (1983)

\section{CONSEIL EXÉCUTIF}

\author{
M. ALEXANDRE HAY, président \\ M. MAURICE AUBERT \\ M. RICHARD PESTALOZZI \\ M. ATHOS GALLINO \\ M. RUDOLF JÄCKLI \\ M"1: ANDREE WEITZEL \\ M. OLIVIER LONG
}

Le Comité international de la Croix-Rouge (CICR) est, avec la Ligue des Sociétés de la Croix-Rouge et du Croissant-Rouge et les 131 Sociétés nationales de la Croix-Rouge et du Croissant-Rouge reconnues, l'une des trois composantes de la Croix-Rouge internationale.

Institution humanitaire indépendante, le CICR est l'organe fondateur de la Croix-Rouge. Intermédiaire neutre en cas de conflits armés et de troubles, il s'efforce d'assurer, de sa propre initiative ou en se fondant sur les Conventions de Genève, protection et assistance aux victimes des guerres internationales et civiles, des troubles et tensions internes, apportant ainsi sa contribution à la paix dans le monde. 


\section{REVUE INTERNATIONALE DE LA CROIX-ROUGE}

\section{SOMMAIRE}

COMITE INTERNATIONAL DE LA CROIX-ROUGE

\section{DANS LE MONDE DE LA CROIX-ROUGE}

FAITS ET DOCUMENTS

LIVRES ET REVUES
ISSN 0035-3361

\section{JANVIER-FÉVRIER 1984 - № 745}

Alexandre Hay : Le CICR et les questions humanitaires internationales

Jean-Pierre Hocké: Action humanitaire: protection et assistance . . . . . . . . . .

Hans-Peter Gasser: Quelques réflexions sur l'avenir du droit international humanitaire

Etats parties aux Conventions de Genève et aux Protocoles additionnels . . . . . . . .

Appel du CICR à l'occasion de l'entrée en vigueur de la Convention sur l'interdiction ou la limitation de l'emploi de certaines armes classiques .............. 31

Démissions et nominations au CICR . . . . . 32

Décès de $\mathbf{M}^{\text {me }}$ M. Gautier-van Berchem. . . . 33

Adhésion aux Protocoles de la République populaire du Congo . . . . . . . . . .

Adhésion de la République arabe syrienne au Protocole I . . . . . . . . . . . . . . . . 34

Adhésion de la Bolivie aux Protocoles. . . . . 35

Adhésion de Costa Rica aux Protocoles . . . . 35

Création d'un Fonds spécial en faveur des handicapés . . . . . . . . . . . . .

Activités extérieures:

Afrique - Amérique latine - Asie - Moyen-

Orient

Conférence des Sociétés nordiques de la Croix-

Rouge . . . . . . . . . . . . . . . 52

Modifications dans le Règlement des Radiocommunications concernant les moyens de transport sanitaires et les moyens de transport neutres............. 54

Le service médical parmi les réfugiés . . . . 64 
La Revue internationale de la Croix-Rouge est publiée par le Comité international de la Croix-Rouge (CICR) depuis 1869. Elle a porté à l'origine le titre de «Bulletin international des Sociétés de secours aux militaires blessés 》, puis de « Bulletin international des Sociétés de la Croix-Rouge ».

- Organe officiel du CICR et de la Croix-Rouge internationale,

- publication spécialisée dans le droit international humanitaire et la doctrine du CICR,

- chronique des activités internationales de la Croix-Rouge, ouvrage de référence essentiellement, qui garde la mémoire des événements,

la Revue internationale maintient un courant d'informations et constitue le lien nécessaire entre les membres de la Croix-Rouge internationale.

La Revue internationale de la Croix-Rouge paraît, une fois tous les deux mois, en trois éditions principales:

en français: Revue internationale de LA CROIX-Rouge

en anglais: International Review of the Red Cross (depuis 1961)

en espagnol: Revista Internacional de la Cruz Roja (depuis 1976)

Elle publie également, en allemand, un cahier d'Extraits des textes parus dans les éditions principales.

RÉDAction: Michel Testuz

ADREsSE: Revue internationale de la Croix-Rouge

17, avenue de la Paix

CH - 1211 - Genève, Suisse

AbONNEMENTs: un an 30 francs suisses; le numéro 5 francs.

Extraits en allemand: un an 10 francs; le numéro 2 francs.

Compte de chèques postaux: 12 - 1767 Genève

Compte bancaire: 129.986 Société de Banque Suisse, Genève

Seuls les textes signés par le Comité international de la Croix-Rouge engagent la responsabilité de celui-ci. 